\title{
Uso de anfetaminas por motoristas de caminhão em rodovias do Estado de São Paulo: um risco à ocorrência de acidentes de trânsito?
}

\author{
Amphetamine use by truck drivers on highways of Sao Paulo \\ State: a risk for the occurrence of traffic accidents?
}

\author{
Juliana Takitane ${ }^{1}$ \\ Lucio Garcia de Oliveira ${ }^{1}$ \\ Ligia Góes Endo ${ }^{1}$ \\ Keziah Cristina Barbosa Gruber de Oliveira ${ }^{2}$ \\ Daniel Romero Muñoz ${ }^{1}$ \\ Mauricio Yonamine ${ }^{3}$ \\ Vilma Leyton ${ }^{1}$
}

${ }^{1}$ Departamento de Medicina Legal, Ética Médica e Medicina Social e do Trabalho, Faculdade de Medicina da Universidade de São Paulo. Av. Dr. Arnaldo 455, Cerqueira César. 01246-903 São

Paulo SP.

julianatakitane@gmail.com

${ }^{2}$ Departamento de Polícia

Rodoviária Federal.

${ }^{3}$ Departamento de Análises

Clínicas e Toxicológicas da

Faculdade de Ciências

Farmacêuticas,

Universidade de São Paulo.

\begin{abstract}
The use of amphetamines in Brazil is common among truck drivers, which may be an important factor in the occurrence of traffic accidents. This article seeks to estimate the prevalence of amphetamine use among truck drivers. Drivers $(N=134)$ were stopped on two different highways in Sao Paulo state and they were asked to answer a questionnaire and provide a urine sample for toxicological analysis. All data were analyzed on Stata 8.0. All participants were males with low levels of schooling, whose mean age was 40.8 years. The presence of amphetamines was detected in $10.8 \%$ of all urine samples collected, being commonly justified in order to make truck drivers able to maintain their state of awareness. Amphetamine use was detected among truck drivers on Sao Paulo highways. The problem is that when the stimulant effects wear off, sleepiness due to sleep deprivation reduces concentration and good driver performance, making drivers vulnerable to traffic accidents and the related effects.
\end{abstract}

Key words Amphetamines, Traffic accidents, Drug abuse
Resumo No Brasil, é comum o uso de anfetaminas por motoristas de caminhão, o que pode culminar na ocorrência de acidentes de trânsito. $O$ objetivo deste artigo é estimar a prevalência do uso de anfetaminas entre caminhoneiros. Motoristas $(N=134)$ foram abordados em duas rodovias do Estado de São Paulo e solicitados a responder um questionário, assim como a fornecer uma amostra de urina para realização de análises toxicológicas. Todos os dados foram analisados em Stata 8.0. Todos os participantes eram do sexo masculino, de idade média de 40,8 anos e de baixa escolaridade. A presença de anfetaminas foi detectada em 10,8\% das amostras de urina, cujo uso foi justificado para manter a vigília durante o trabalho. O uso de anfetaminas foi detectado entre caminhoneiros em rodovias de São Paulo. Cessado o efeito estimulante, a sonolência advinda de uma possível privação de sono diminui a atenção e o bom desempenho na direção, predispondo o condutor aos acidentes de trânsito e seus custos relacionados.

Palavras-chave Anfetaminas, acidentes de trânsito, drogas de abuso 


\section{Introdução}

No Brasil, o sistema de transporte de cargas é o principal meio utilizado para a movimentação da economia no país, contando, para isso, com aproximadamente quase dois milhões de quilômetros de rodovias ${ }^{1}$, localizadas principalmente na região sudeste do país, especialmente no Estado de São Paulo, cujos 200 mil km de estradas são utilizados para o transporte de mais de $90 \%$ de toda produção final e serviços do país².

Entretanto, desperta a atenção o número de acidentes de trânsito (AT) nas rodovias brasileiras. Nesse sentido, um relatório recentemente lançado pelo Departamento Nacional de Infraestrutura de Transportes (DNIT) apontou para a ocorrência de 320 mil acidentes rodoviários no país, dos quais $35,1 \%$ aconteceram na região sudeste, tendo o Estado de São Paulo respondido por uma prevalência de $20,4 \%{ }^{3}$. Ainda no Estado de São Paulo, os veículos de carga responderam por mais de $30 \%$ desses acidentes ${ }^{3}$, embora tenham apenas $9 \%$ da frota nacional de veículos automotores ${ }^{4}$.

Em termos de etiologia, os AT são fenômenos multicausais, entretanto, o uso de substâncias psicoativas (SPA) é sabidamente fator contribuinte para seu acontecimento ${ }^{5-7}$, de tal forma que a ingestão de qualquer quantidade de SPA é sugerida como capaz de provocar alterações cognitivas que não são condizentes com o desempenho de uma direção segura, seja através de seus efeitos agudos, seja por meio de seus efeitos residuais ou de abstinência ${ }^{8,9}$.

De fato, alguns estudos brasileiros têm demonstrado que a classe dos motoristas profissionais de caminhão tem estado susceptível ao uso de SPA, um comportamento que pode ser empregado como forma de alívio às condições inadequadas de seu trabalho, seja em decorrência das longas jornadas, da pressa para chegar ao local de destino, baixa remuneração, poucas horas de sono e baixa qualidade de saúde e de vida como um todo ${ }^{10-14}$, assim como para o manejo dos efeitos desagradáveis advindos de possíveis transtornos do humor instalados ${ }^{15}$.

Portanto, as anfetaminas - popularmente conhecidas como "rebites", potentes estimulantes do Sistema Nervoso Central (SNC) - estão entre as SPA comumente utilizadas por esses trabalhadores. Embora esse efeito possa ser entendido como uma vantagem ao condutor, à medida que a concentração plasmática de anfetaminas aumenta, menor é o desempenho do condutor na direção ${ }^{16}$. Sobretudo, preocupa o fato de que, finalizado o efeito estimulante das anfetaminas, o condutor estará submetido a um efeito "rebote" sobre o SNC, que envolve a indução de depressão, fadiga e sono ${ }^{17-19}$. Assim, em conjunto, os efeitos agudos e residuais das anfetaminas submetem o condutor a situações de risco no trânsito, impedindo-o de realizar uma direção considerada segura. A periculosidade dessa associação entre motoristas de caminhão foi apontada por um estudo brasileiro que encontrou que $27 \%$ dos entrevistados relataram envolvimento em acidentes nas estradas devido ao uso de anfetaminas ${ }^{12}$.

Embora o uso de "rebites" por motoristas de caminhão seja de conhecimento popular, poucos foram os estudos brasileiros desenvolvidos no sentido de estimar a prevalência desse uso, assim como de outras SPA. A saber, esse tipo de quantificação pode ser adquirido, com sucesso, através da análise toxicológica de espécimes biológicos (saliva e urina). Entretanto, poucos foram os estudos brasileiros que adotaram essa metodologia ${ }^{10,14,20}$, assim como poucos são os baseados em relato ${ }^{11,12}$.

Logo, acreditamos que o conhecimento da prevalência do uso de anfetaminas por motoristas de caminhão é uma informação imprescindível para o melhor conhecimento da magnitude do problema em nosso país, bem como para fornecer dados às autoridades públicas e competentes para planejar e implementar ações no sentido de melhorar as condições de trabalho dessa população, prevenindo o uso de drogas, o acontecimento de AT e seus desdobramentos a curto, médio e longo-prazo. Sobretudo, a importância dessa informação é que as consequências dos AT têm ultrapassado a esfera individual, afetando a sociedade como um todo. Assim sendo, o presente estudo teve por objetivo estimar a prevalência do uso de anfetaminas entre motoristas de caminhão que trafegavam por duas rodovias do estado de São Paulo (Régis Bittencourt e Transbrasiliana) através da realização de análises toxicológicas em urina e aplicação de questionário.

\section{Métodos}

O método foi exploratório-descritivo e de corte transversal. As amostras de urina e outros dados foram coletados durante duas edições do projeto "Comandos de Saúde nas Rodovias", do Departamento de Polícia Rodoviária Federal (DPRF), realizados durante o ano de 2010. Este projeto do DPRF tem o objetivo de orientar os profissionais de transporte sobre bons hábitos 
de saúde e detectar fatores de risco à saúde, como pressão alta, obesidade, estresse e sonolência ${ }^{21}$.

\section{Casuística}

Utilizou-se uma amostra de conveniência composta por 134 motoristas de caminhão que circulavam pelas rodovias Régis Bittencourt (BR 116), Km 296, em Itapecerica da Serra e Transbrasiliana (BR 153), Km 259, em Marília, ambas no estado de São Paulo. As cidades de Itapecerica da Serra e Marília abrangem importantes regiões para a rota de tráfego de pessoas e cargas, não apenas para o estado, mas também na interligação de diferentes regiões do país. Os participantes que circulavam por essas rodovias foram abordados por policiais rodoviários e convidados a participar do projeto "Comandos de Saúde nas Rodovias", acima mencionado. Desse total, quatro (3\%) dos sujeitos abordados recusaram a participar do estudo, totalizando a participação de 130 motoristas de caminhão.

\section{Instrumento de pesquisa}

Um instrumento de pesquisa estruturado (constituído por 46 perguntas) foi elaborado com fins de registrar as informações pessoais (idade, sexo, estado civil, etnia, nível de escolaridade, classe socioeconômica, entre outras), ocupacionais (tipo de vínculo empregatício - se contratado ou autônomo; duração da jornada de trabalho diária; local de partida e destino; número de horas já viajadas e a viajar, entre outras) e de saúde dos participantes. Os participantes também foram solicitados a responder sobre a experiência pessoal em relação ao uso de álcool, tabaco e outras drogas, especialmente de anfetaminas.

\section{Procedimentos}

Após terem sido esclarecidos sobre os objetivos do estudo e do sigilo das informações, os participantes foram solicitados a assinar o Termo de Consentimento Livre e Esclarecido (TCLE) para então responderem ao instrumento de pesquisa. A participação foi finalizada através do fornecimento de uma amostra de urina, coletada por micção espontânea, em frascos de polietileno previamente identificados com um registro de controle laboratorial, preservando assim a confidencialidade dos sujeitos de pesquisa. As amostras coletadas foram mantidas sob refrigeração em geladeiras térmicas e conduzidas ao laboratório no mesmo dia da coleta (período não superior a seis horas), tendo sido armazenadas em freezer $\left(-20^{\circ} \mathrm{C}\right)$. Posteriormente, essas amostras foram descongeladas à temperatura ambiente à medida que foram procedidas as análises toxicológicas. Um período máximo de seis dias foi permitido entre a coleta e a análise das amostras de urina.

\section{Análise toxicológica}

As amostras de urina foram submetidas à triagem de anfetaminas por imunoensaio utilizando o teste On-Site ${ }^{\circledR} \mathrm{CupKit}^{\mathrm{TM}} 5_{01}$, Varian. As amostras que apresentaram resultados positivos no teste de triagem foram então submetidas às análises confirmatórias empregando-se a técnica de cromatografia gasosa acoplada à espectrometria de massas (CG-EM) (GC 5890/MS 5975 Agilent), utilizando método previamente publicado $^{22}$. Os seguintes valores de referência (cutoffs) foram utilizados para a detecção de anfetaminas na urina: (a) etapa de triagem $=1.000 \mathrm{ng}$ / $\mathrm{mL}$ e (b) análise confirmatória $=500 \mathrm{ng} / \mathrm{mL}$.

\section{Análise estatística}

Foi utilizada estatística descritiva das informações sociodemográficas, ocupacionais, de saúde e do uso de drogas utilizando-se o programa Stata 8.0 (Stata Corp., College Station, Estados Unidos). Os resultados foram descritos na forma de prevalência (\%) e intervalo de confiança 95\% (IC95\%).

\section{Aspectos éticos}

O protocolo de pesquisa foi aprovado pela Comissão de Ética para Análise de Projetos de Pesquisa do HC-FMUSP.

\section{Resultados}

Todos os motoristas eram do sexo masculino, com idade média de 40,8 anos (IC95\%: 39,0-42,7 anos). A maioria se considerou dentro de um relacionamento estável, sendo que $70 \%(62,1$ $77,9)$ declararam-se casados. Mais da metade dos participantes $(65,5 \%)$ declarou ter até oito anos de escolaridade. Em relação às informações ocupacionais, o tempo médio relatado de profissão foi de 16,7 anos $(14,8-18,5)$ e $68 \%$ dos participantes era previdenciário (59,7-75,7) (Tabela 1).

Sobre as informações de saúde, 20\% dos entrevistados tinha pelo menos um problema de 
saúde, sendo que desses, $12,3 \%$ relataram ter pressão alta $(6,7-18,0), 7,7 \%$ estresse $(3,1-12,3)$, $4,6 \%$ diabetes $(1,0-8,2)$ e $30,8 \%(22,8-38,7)$ queixaram-se de alguma dor, sendo a região lombar

Tabela 1. Informações sociodemográficas e ocupacionais entre motoristas de caminhão abordados em duas rodovias do Estado de São Paulo, Brasil, 2011 ( $\mathrm{N}=130)$.

\begin{tabular}{lrr}
\hline \multicolumn{1}{c}{ Variável } & Média & \multicolumn{1}{c}{ IC 95\% } \\
\hline Idade (em anos) & 40,8 & $39,0-42,7$ \\
Tempo de profissão (em anos) & 16,7 & $14,8-18,6$ \\
& & \\
& $\%$ & \multicolumn{1}{c}{ IC 95\% } \\
Estado Civil & & \\
$\quad$ Solteiro & 20,0 & $13,1-26,9$ \\
Casado & 70,0 & $62,1-77,9$ \\
Amasiado & 4,6 & $1,0-8,2$ \\
Divorciado & 4,6 & $1,0-8,2$ \\
Viúvo & 0,8 & $0,0-2,3$ \\
Escolaridade (em anos) & & \\
$\quad$ Até 4 & 18,5 & $11,8-25,1$ \\
De 5 a 8 & 46,9 & $38,3-55,5$ \\
De 9 a 11 & 26,2 & $18,6-33,7$ \\
Superior a 11 & 8,5 & $3,7-13,2$ \\
Tipo de serviço & & \\
Autônomo & 32,3 & $24,3-40,3$ \\
Previdenciário & 67,7 & $59,7-75,7$ \\
Aposentado & 0,8 & $0,0-2,3$ \\
Total & $\mathbf{1 3 0}$ & $\mathbf{1 3 0}$ \\
\hline
\end{tabular}

$(14,6 \% ; 8,5-20,7)$ e cervical $(10 \% ; 4,8-15,2)$ as mais frequentemente referidas. No que diz respeito ao uso de drogas, 66,2\% (58,0-74,3) declararam-se não fumantes, $10,8 \%$ ex-fumantes $(5,4-16,1)$ e, finalmente, $23,1 \%(15,8-30,3)$ fumantes. Neste último grupo, o tempo médio de uso de tabaco foi de 16,1 anos (12,7-19,6). Ainda, 69,2\% (61,3$77,2)$ dos participantes relataram fazer uso de álcool - sendo que 95,6\% (91,3-99,8) deles citaram a cerveja como a bebida de preferência - e $33,0 \%(25,2-41,9)$ relataram já ter feito uso de anfetaminas (Tabela 2). Em relação às análises toxicológicas, $10,8 \%(5,4-16,1)$ das amostras de urina apresentaram resultado positivo para anfetamina (Tabela 2), dos quais $6,2 \%$ na rodovia Régis Bittencourt e 4,6\% na rodovia Transbrasiliana. O produto mais citado foi o Desobesi ${ }^{\circledR}$. A justificativa relatada para esse uso foi para mantê-los acordados, a fim de cumprir os prazos de entrega da carga transportada.

Os dados de relato sobre o uso de anfetaminas foram analisados para avaliar se os resultados do discurso e da análise toxicológica eram concordantes. Assim, apenas seis dos motoristas $(42,9 \% ; 16,9-68,8)$ cuja amostra de urina foi positiva para o uso de anfetaminas declararam ter feito esse uso dentro do prazo de até cinco dias, intervalo de tempo em que o uso é detectável na urina (Tabela 3 ).

Tabela 2. Informações de saúde e do uso de drogas entre motoristas de caminhão abordados em duas rodovias do Estado de São Paulo, Brasil, 2011 ( $\mathrm{N}=130)$.

\begin{tabular}{|c|c|c|c|c|c|}
\hline $\begin{array}{l}\text { Variável } \\
\text { Por relato }\end{array}$ & $\%$ & IC $95 \%$ & $\begin{array}{l}\text { Variável } \\
\text { Por relato }\end{array}$ & $\%$ & IC $95 \%$ \\
\hline Número de problemas de saúde & & & Tabaco & & \\
\hline 0 & 80,0 & $73,1-86,9$ & Não & 66,2 & $58,0-74,3$ \\
\hline 1 & 16,2 & $9,8-22,5$ & Sim & 23,1 & $15,8-30,3$ \\
\hline 2 & 3,1 & $0,1-6,0$ & Tempo de uso (em anos) & 16,1 & $12,7-19,6$ \\
\hline 3 & 0,8 & $0,0-2,3$ & Ex-fumante & 10,8 & $5,4-16,1$ \\
\hline Pressão Alta & 12,3 & $6,7-18,0$ & Álcool & & \\
\hline Diabetes & 4,6 & $1,0-8,2$ & Sim & 69,2 & $61,3-77,2$ \\
\hline Estresse & 7,7 & $3,1-12,3$ & Cerveja & 95,6 & $91,3-99,8$ \\
\hline Dores & 30,8 & $22,8-38,7$ & Destilado & 7,8 & $2,2-13,3$ \\
\hline Ombro & 6,9 & $2,6-11,3$ & Vinho & 10,0 & $3,8-16,2$ \\
\hline Cervical & 10,0 & $4,8-15,2$ & Anfetaminas & 33,0 & $25,2-41,9$ \\
\hline Joelho & 1,5 & $0,0-3,7$ & \multirow{2}{*}{\multicolumn{3}{|c|}{ Resultado de Toxicologia }} \\
\hline Lombar & 14,6 & $8,5-20,7$ & & & \\
\hline \multirow[t]{3}{*}{ Total } & 130 & & Anfetamina & 10,8 & $5,4-16,1$ \\
\hline & & & Usou nos últimos cinco dias & 42,9 & $16,9-68,8$ \\
\hline & & & Total & 130 & \\
\hline
\end{tabular}




\section{Discussão}

Socialmente, apesar de não se ter conhecimento de uma "epidemia" de uso de anfetaminas no Brasil, seu uso recreacional tem sido acompanhado por uma série histórica de levantamentos estatísticos conduzidos em diversificados segmentos sociais. Nesse sentido, o "II Levantamento Domiciliar sobre o Uso de Drogas no Brasil: Estudo envolvendo as 108 maiores cidades do país" apontou que $22,8 \%$ da população geral brasileira acima de 12 anos de idade declararam já ter feito o uso ilícito e experimental de pelo menos uma substância psicotrópica (exceto álcool e tabaco), dos quais $3,2 \%$ relataram ter feito o uso de estimulantes (moderadores de apetite; anfetaminas) em algum momento da vida, o que equivale a uma população de quase 1.6 milhões de brasileiros ${ }^{23}$. Já o "VI Levantamento Nacional sobre o Consumo de Drogas Psicotrópicas entre Estudantes de Ensino Fundamental e Médio das Redes Pública e Privada de Ensino nas 27 Capitais Brasileiras", conduzido com 50.890 estudantes, apontou que $2,2 \%$ deles já usaram anfetamínicos em algum momento da vida, um uso que vem diminuindo em 25 anos da realização desse tipo de estudo nesse segmento social ${ }^{24}$. Por outro lado, a prevalência desse uso é bem maior entre estudantes universitários conforme apontado pelo "I Levantamento Nacional sobre o Uso de Álcool, Tabaco

e Outras Drogas entre Universitários das $27 \mathrm{Ca}-$ pitais Brasileiras". Assim, em uma amostra populacional de 12.711 universitários, $13,8 \%$ deles relataram já ter feito uso recreacional de anfetaminas na vida, especialmente entre as mulheres com idade superior a 35 anos $^{25}$. Na verdade, esse dado reflete a cultura brasileira no que se refere ao uso de anfetaminas. Nesse sentido, a análise de prescrições obtidas de farmácias de manipulação e drogarias indicavam que as mulheres (especialmente as de meia-idade) recebiam a maioria das prescrições para anorexígenos tipo anfetamina, um uso com fins estéticos ${ }^{26}$. Também, o uso de anfetaminas já foi observado na prática esportiva, dado que - por serem estimulantes do SNC aumentam a resistência à fadiga e melhoram o desempenho, a exemplo do que acontece em esportes de modalidade de luta. Assim, um levantamento realizado no ano de 2003 apontou que de um total de 3.266 atletas brasileiros em competição, seis deles haviam feito uso de estimulantes incluindo-se, neste grupo, as anfetaminas ${ }^{27}$.

Entretanto, entre os diversos segmentos sociais, é o uso de drogas entre condutores de veículos automotivos que merece especial atenção, pois já é de consenso que o uso de álcool, drogas ilícitas e medicamentos podem prejudicar o ato de dirigir, aumentando assim o risco da ocorrência de $\mathrm{AT}^{21,28,29}$. A situação parece ser ainda mais preocupante entre os motoristas de caminhão, uma população móvel que se distribui por todo o país, uma vez que, como observado aqui neste estudo, $10,8 \%$ dos participantes tinha feito uso de anfetaminas conforme os resultados de análises toxicológicas conduzidas em urina.

No Brasil, alguns dados sobre o uso de anfetaminas por motoristas de caminhão foram obtidos através de relato e de análises toxicológicas realizadas em matrizes biológicas coletadas desses profissionais. A exemplo, Souza et al. ${ }^{11}$, em 2005 , relataram o uso de anfetaminas por $11,1 \%$ dos motoristas de caminhão entrevistados. Já Nascimento et al..$^{12}$, em 2007, obtiveram uma prevalência de uso bem superior, ao terem apontado que $66 \%$ dos motoristas de caminhão relataram estar acostumados a fazer uso de anfetaminas durante suas viagens. Por outro lado, outros estudos estimaram o uso de anfetaminas através de análises toxicológicas de amostras de urina. Em 2003, Silva et al. ${ }^{20}$ realizaram uma pesquisa com 728 amostras de urina de motoristas de caminhão abordados em rodovias em três regiões do Brasil, dos quais 4,8\% eram casos positivos para anfetaminas. Outro estudo apontou, também através de análises toxicológicas na 
urina, uma prevalência de 7,0\% de uso de drogas entre motoristas de caminhão que trafegavam em estradas do estado de São Paulo, sendo, nesses casos $18 \%$ para anfetaminas ${ }^{10}$. Mais recentemente, Leyton et al. ${ }^{14}$ apontaram que 5,7\% dos motoristas de caminhão abordados, também em rodovias paulistas, apresentaram resultado positivo na urina para a presença de anfetaminas.

Seja como for, os resultados ainda são controversos, uma vez que os achados são divergentes entre estudos e, especialmente, entre os diferentes meios utilizados para o levantamento de informação, seja por relato ou análise toxicológica de urina. Nesse sentido, no presente estudo, pouco mais de $40 \%$ dos motoristas de caminhão cuja amostra de urina foi positiva para anfetaminas, declararam realmente ter feito esse uso dentro do período de tempo em que a substância pode ser detectada na urina (até cinco dias após o consumo $)^{30}$. De qualquer forma, a avaliação do uso de álcool e drogas por relato tem sido apontada como confiável por estudos prévios. Nesse sentido, aponta-se que a confiabilidade da resposta de dependentes químicos (sobre o próprio uso de drogas) é confiável, especialmente na presença de fatores tais como tempo curto de entrevista, simplicidade das questões e prévio treinamento do entrevistador ${ }^{31}$, condições que acreditamos terem sido satisfeitas por nossa pesquisa. Um estudo mais recente também apontou para a confiabilidade das informações sobre o uso de drogas quando obtidas por relato, mesmo na condição de avaliação de um padrão de uso realizado a longo-prazo ${ }^{32}$. Entretanto, os possíveis motivos de discordância entre os resultados de relato e os resultados de análise toxicológica não serão aqui pormenorizados, mas ressaltamos a necessidade de incentivar a realização de mais estudos que possam abordar essa questão em outras condições que as diferentes do trabalho de campo que originou este manuscrito.

Também merece destaque que o Desobesi ${ }^{\circledast}$ foi o medicamento mais frequentemente usado entre os participantes, um anorexígeno que tem como princípio ativo o cloridrato de femproporex. Assim como os demais derivados anfetamínicos, promove a estimulação do SNC e apresenta elevado potencial para causar dependência. É biotransformado em anfetamina no organismo humano, de tal forma que os resultados dos exames toxicológicos que apontaram a presença deste produto nas amostras de urina deviam ter como origem a ingestão de femproporex ${ }^{33}$.

Em relação ao uso de anfetaminas no país, vale ressaltar que no dia 6 de outubro de 2011, a
Agência Nacional de Vigilância Sanitária - ANVISA implementou a Resolução RDC 52/2011 que vetou a fabricação, a importação, a exportação, a distribuição, a manipulação, a prescrição, a dispensação, o aviamento, o comércio e o uso de medicamentos ou fórmulas medicamentosas que contenham as substâncias anfepramona, femproporex e mazindol, seus sais e isômeros, bem como intermediários $^{34}$. Assim, daqui pra frente, outros estudos serão necessários no sentido de avaliar o impacto da lei sobre a oferta e o uso de anfetaminas pelos motoristas de caminhão nas estradas, assim como uma possível transição para outras drogas estimulantes do SNC, o que sugerimos que seja feito especialmente a partir de análise toxicológica de espécimes biológicos, uma metodologia que incentivamos que seja usada por estudos futuros.

Também, aparte dos resultados sobre a prevalência, aqui merecem ser discutidos os motivos que têm levado essa categoria profissional a se engajar no uso de anfetaminas, assim como sua repercussão. Então, entre esses trabalhadores, seu uso tem sido justificado com fins de reduzir o sono, aumentar a jornada, o rendimento e a produtividade no trabalho ${ }^{14}$, o que os leva a cumprir uma jornada de até 16 horas diárias, realizar o trabalho em turnos, assim como ter um período médio de sono de apenas 5 horas diárias ${ }^{11}$. De fato, estudos realizados em simuladores têm descrito que pequenas doses de anfetaminas possam melhorar certas funções cognitivas e, consequentemente, o desempenho na direção ${ }^{16}$. No entanto, acredita-se - por relatos informais - que o uso de anfetaminas entre esses profissionais não seja um uso leve. Nesse sentido, é preocupante que o uso excessivo de anfetaminas tenha o efeito oposto ao desejado, já tendo sido descrita uma relação positiva entre o aumento da concentração sanguínea de anfetaminas e a piora de desempenho na direção ${ }^{16}$.

Assim, testemunha-se que o uso de drogas por esses profissionais tem sido mantido como uma forma de garantir um melhor ajustamento à má qualidade de trabalho a que estão comumente submetidos, um comportamento que carrega consigo uma série de potenciais desdobramentos negativos, seja à saúde do próprio condutor, seja à segurança nas vias públicas e à economia do país, refletindo na sociedade como um todo. Deste modo, concluindo, consideramos que os dados do presente trabalho possam contribuir para a implementação de políticas públicas que possam melhorar - ou reverter - a situação aqui descrita, promovendo subsídios para a re- 
gulamentação da jornada de trabalho da categoria, assim como a elaboração de estratégias de prevenção que evitem o uso de drogas entre esses profissionais, e também a sua progressão a outros psicoestimulantes de maior potencial de dependência. Finalmente, incentivamos a realização de uma pesquisa que possa descrever a situação em uma amostra representativa do território nacional.

\section{Colaboradores}

J Takitane trabalhou desde a concepção do manuscrito até sua redação propriamente dita; LG Oliveira auxiliou na análise, interpretação dos dados e redação do manuscrito; LG Endo e KCBG Oliveira auxiliaram no levantamento dos dados durante o trabalho de campo da pesquisa; DR Muñoz auxiliou na redação e revisão do manuscrito; M Yonamine auxiliou na análise toxicológica dos espécimes biológicos, assim como na redação do manuscrito; V Leyton auxiliou na redação e revisão do manuscrito.

\section{Agradecimentos}

Os autores agradecem aos policiais do DPRF que auxiliaram na coleta dos dados; à FUNDAP pela bolsa concedida à primeira autora durante estágio no programa de aprimoramento do Hospital das Clínicas da Faculdade de Medicina da Universidade de São Paulo - HC-FMUSP e ao LIM-40-HCFMUSP pela possibilidade de realização do estudo.

\section{Referências}

1. Departamento de Informática do SUS (DATASUS). Informações de Saúde - Epidemiológica e Morbidade. [página da Internet]. [acessado 2010 jun 08]. Disponível em: http://tabnet.datasus.gov.br/cgi/deftohtm. exe?idb2008/c09.def

2. Instituto de Pesquisa Econômica Aplicada (IPEA). Impactos sociais e econômicos dos acidentes de trânsito em aglomerações urbanas brasileiras. [documento na Internet]. [acessado 2010 jul 17]. Disponível em: http://www.scribd.com/doc/17316648/Ipea-Relatorio -Executivo

3. Departamento Nacional de Infraestrutura de Transportes (DNIT). Anuário Estatístico das Rodovias Federais 2010: Acidentes de Trânsito e Ações de Enfrentamento ao Crime. [documento na Internet]. [acessado 2010 jul 17]. Disponível em: http://www. dnit. gov.br/rodovias/operacoes-rodoviarias/estatisticasde-acidentes/anuario-2010.pdf

4. São Paulo. Governo do Estado de São Paulo. Secretaria dos Transportes. [página da Internet]. [acessado 2010 set 20]. Disponível em: http://www.transportes. sp.gov.br/v20/noticias/imprime_noticia.asp?cod $=2105$

5. De Boni R, Bozzetti MC, Hilgert J, Sousa T, Von Diemen L, Benzano D, Menegon G, Holmer B, Duarte Pdo C, Pechansky F. Factors associated with alcohol and drug use among traffic crash victims in southern Brazil. Accid Anal Prev 2011; 43(4):14081413.

6. Arnold PK, Hartley LR, Corry A, Hochstadt D, Penna F, Feyer AM. Hours of work and perceptions of fatigue among truck drivers. Accid Anal Prev 1997; 29(4):471-477.

7. Dinges DF. An overview of sleepiness and accidents. J Sleep Res 1995; 4(S2):4-14.

8. Moskowitz H, Burns MM, Williams AF. Skills performance at low blood alcohol levels. J Stud Alcohol 1985; 46(6):482-485.

9. Hingson R, Winter M. Epidemiology and consequences of drinking and driving. Alcohol Res Health 2003; 27(1):63-78. 
10. Yonamine M. A saliva como espécime biológico para monitorar o uso de álcool, anfetamina, metanfetamina, cocaina e maconha por motoristas profissionais [tese]. São Paulo (SP): Faculdade de Ciências Farmacêuticas, Universidade de São Paulo; 2004.

11. Souza JR, Paiva T, Reimão R. Sleep habits, sleepiness and accidents among truck drivers. Arq Neuropsiquiatr 2005; 63(4):925-930.

12. Nascimento EC, Nascimento E, Silva JP. Uso de álcool e anfetaminas entre caminhoneiros de estrada. Rev Saude Publica 2007; 41(2):290-293.

13. Masson VA, Monteiro MI. Vulnerabilidade à doenças sexualmente transmissíveis/AIDS e uso de drogas psicoativas por caminhoneiros. Rev Bras Enferm 2010; 63(1):79-83.

14. Leyton V, Sinagawa DM, Oliveira KCBG, Schmitz W, Andreuccetti G, De Martinis BS, Yonamine M, Muñoz DR. Amphetamine, cocaine and cannabinoids use among truck drivers on the roads in the State of Sao Paulo, Brazil. Forensic Sci Int 2012; 215(1-3):25-27.

15. da Silva-Júnior FP, de Pinho RS, de Mello MT, de Bruin VM, de Bruin PF. Risk factors for depression in truck drivers. Soc Psychiatry Psychiatr Epidemiol 2009; 44(2):125-129.

16. Musshoff F, Madea B. Driving under the influence of amphetamine-like drugs. J Forensic Sci 2012; 57(2):413-419.

17. Ponce JC, Leyton V. Drogas ilícitas e trânsito: problema pouco discutido no Brasil. Rev Psiq Clín 2008; 35(1):65-69.

18. Rozestraten RJA. Psicologia do trânsito: conceitos e processos básicos. São Paulo: EPU-EDUSP; 1988.

19. United Nations Office on Drugs and Crime (UNODC). Vienna: Preventing amphetamine-type stimulant use among young people. A policy and programming guide. [documento na Internet]. [acessado 2010 nov 11]. Disponível em: http://www. unodc.org/pdf/youthnet/ATS.pdf

20. Silva OA, Greve JMD, Yonamine M, Leyton V. Drug use by truck drives in Brazil. Drugs Educ Prev Pol 2003; 10(2):135-139.

21. Sest Senat. Comandos de Saúde nas Rodovias. [página da Internet]. [acessado 2010 jul 24]. Disponível em: http://www.sestsenat.org.br/Paginas/Comandos-deSaude.aspx

22. Scheidweiler KB, Huestis MA. A validated gas chromatographic-electron impact ionization mass spectrometric method for methylenedioxymethamphetamine (MDMA), methamphetamine and metabolites in oral fluid. J Chromatogr B Analyt Technol Biomed Life Sci 2006; 835(1-2):90-99.

23. Carlini EA, Galduroz JCF. Levantamento domiciliar sobre o uso de drogas psicotrópicas no Brasil: estudo envolvendo as 108 maiores cidades do país. São Paulo: CEBRID/UNIFESP; 2007.
24. Carlini EA, Noto AR, Sanchez ZM. VI Levantamento Nacional sobre o Consumo de Drogas Psicotrópicas entre Estudantes de Ensino Fundamental e Médio das Redes Pública e Privada de Ensino nas 27 Capitais Brasileiras - 2010. Brasília: SENAD; 2010.

25. Andrade AG, Duarte PCAV, Barroso LP, Nishimura R, Alberghini DG, Oliveira LG. Alcohol and other drug use among Brazilian college students: Effects of gender and age. Rev Bras Psiquiatr 2012; 34:294-305.

26. Nappo SA, Carlini EA, Araújo MD, Moreira LFS. Prescription of anorectic and benzodiazepine drugs through notification B prescriptions in Natal, Rio Grande do Norte, Brazil. Braz J Pharm Sci 2010; 46(2):297-303.

27. De Rose EH, Neto FRA, Moreau RLM, Castro RRT. Controle antidoping no Brasil: resultados do ano de 2003 e atividades de prevenção. Rev Bras Med Esporte 2004; 10(4):289-293.

28. Brasil. Lei no. 9.503, de 23 de setembro de 1997. Diário Oficial da União 1997; 24 set.

29. Brasil. Resolução no ${ }^{\circ} .81$, de 19 de novembro de 1998. Disciplina o uso de medidores da alcoolemia e a pesquisa de substâncias entorpecentes no organismo humano, estabelecendo os procedimentos a serem adotados pelas autoridades de trânsito e seus agentes. Diário Oficial da União 1998; 25 nov.

30. Moffat AC, Osselton MD, Widdop B. Clarke's anal$y$ sis of drug and poisons. London: Pharmaceutical Press; 2004.

31. Kokkevi A, Richardson C, Palermou B, Leventakou V. Reliability of drug dependents' self-reports. Drug Alcohol Depend 1997; 45(1-2):55-61.

32. Murphy DA, Hser YI, Huang D, Brecht ML, Herbeck DM. Self-report of Longitudinal Substance Use: A Comparison of the UCLA Natural History Interview and the Addiction Severity Index. J Drug Issues 2010; 40(2):495-516.

33. Cody JT, Valtier S. Detection of amphetamine following administration of fenproporex. J Anal Toxicol 1996; 20(6):425-431.

34. Brasil. Resolução no 52 , de 6 de outubro de 2011. Dispõe sobre a proibição do uso das substâncias anfepramona, femproporex e mazindol, seus sais e isômeros, bem como intermediários e medidas de controle da prescrição e dispensação de medicamentos que contenham a substância sibutramina, seus sais e isômeros, bem como intermediários. Diário Oficial da União 2011; 6 out.

Artigo apresentado em 08/11/2012

Aprovado em 10/12/2012

Versão final apresentada em 18/12/2012 